\title{
The Effectiveness of Nasal Airway Stent Therapy for the Treatment of Mild-to-Moderate Obstructive Sleep Apnea Syndrome
}

\author{
Kengo Ohtsuka ${ }^{a}$ Rie Baba ${ }^{a}$ Wakako Yamasawa ${ }^{b}$ Ryutaro Shirahama ${ }^{c}$ \\ Yoichiro Hattori $^{d}$ Hiroshi Senoura ${ }^{\mathrm{e}}$ Tomoko Betsuyaku ${ }^{a}$ Koichi Fukunaga ${ }^{a}$ \\ aDivision of Pulmonary Medicine, Department of Medicine, Keio University School of Medicine, Tokyo, Japan; \\ ${ }^{b}$ Department of Laboratory Medicine, Keio University School of Medicine, Tokyo, Japan; 'RESM Shin Yokohama \\ Sleep \& Respiratory Medical Care Clinic, Kanagawa, Japan; ${ }^{d}$ Kichijyouji Sleep Medical Clinic, Tokyo, Japan; ${ }^{\text {eShinjuku }}$ \\ Sleep Medical Clinic, Tokyo, Japan
}

\section{Keywords}

Alternative therapy · Apnea-Hypopnea Index - Nasal airway stent $\cdot$ Obstructive sleep apnea syndrome $\cdot$ Snoring

\begin{abstract}
Background: Patients with obstructive sleep apnea syndrome (OSAS) experience excessive daytime sleepiness and insomnia and they are at risk of developing cardiovascular disease and stroke. Continuous positive airway pressure therapy could improve symptoms and decrease these risks; however, adherence is problematic. Although the oral appliance is another therapeutic option, patient satisfaction is limited and the effect of the nasal airway stent - a new device - remains unclear. Objectives: The aim of this study was to evaluate the effect of NAS therapy in patients with mild-tomoderate OSAS in a prospective, single-arm, interventional pilot study. Method: Patients with mild/moderate sleep apnea ( $n=71$; Apnea-Hypopnea Index [AHI], 5-20 events/h on polysomnography) were recruited. Sleep-associated events were measured using a portable device (WatchPAT200) preand immediately post-treatment and at 1 month follow-up. $\mathrm{AHI}$ (including supine and non-supine AHI), Oxygen Desaturation Index (ODI), Respiratory Disturbance Index (RDI), percutaneous oxygen saturation, heart rate, and snore volume
\end{abstract}

karger@karger.com www.karger.com/res

Karger"

GOPEN ACCESS
(C) 2020 The Author(s)

Published by S. Karger AG, Basel

This is an Open Access article licensed under the Creative Commons Attribution-NonCommercial-4.0 International License (CC BY-NC) (http://www.karger.com/Services/OpenAccessLicense), applicable to the online version of the article only. Usage and distribution for commercial purposes requires written permission. were evaluated. Symptoms were assessed using the Epworth Sleepiness Scale, Pittsburgh Sleep Quality Index, and Hospital Anxiety and Depression Scale. Results: NAS use significantly improved $\mathrm{AHI}$, supine $\mathrm{AHI}, \mathrm{RD}, \mathrm{ODI}$, and snore volume compared to pre-intervention $(r=0.44,0.48,0.3,0.42$, and 0.34; $p<0.001, p<0.001, p=0.011, p<0.001$, and $p=$ 0.048 , respectively). Additionally, 25 and $10 \%$ of patients showed complete and partial response for $\mathrm{AHI}$, respectively; these improvements remained significant 1 month later. Pittsburgh Sleep Quality Index scores improved from 6.0 to $5.3(r=0.46, p=0.022)$. Conclusions: NAS therapy reduced severity and snoring in patients with mild-to-moderate OSAS. Approximately $30 \%$ of patients did not tolerate NAS due to side effects. (c) 2020 The Author(s)

Published by S. Karger AG, Basel

\section{Introduction}

Obstructive sleep apnea syndrome (OSAS) is a major cause of sleep-disordered breathing and is characterized by collapse of the upper airway [1]. Because of intermittent hypoxemia [2], OSAS causes unpleasant symptoms, including excessive daytime sleepiness, insomnia [3], morning headache [4], depression [5], and gastroesoph- 
Fig. 1. Features of the NAS. a The NAS comprises a tube-shaped shaft made from rubber and a proximal nose clip to fix the device. $\mathbf{b}$ The NAS is inserted into one of the narises until it reached the retropalatal oropharynx. NAS, nasal airway stent.

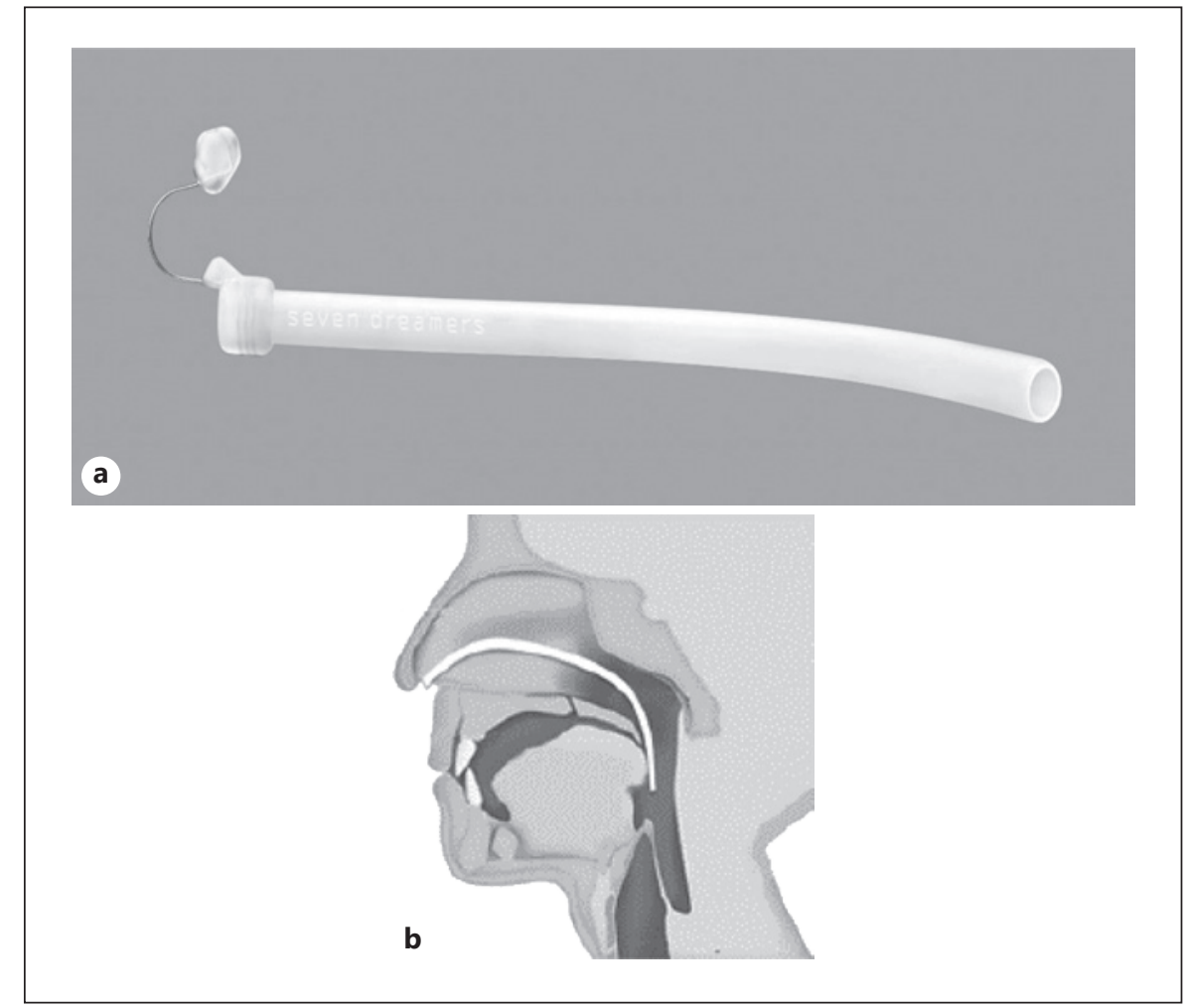

ageal reflux disease [6], and increases the risk of developing some cancer types, cardiovascular diseases, and stroke [7-13]. The American Academy of Sleep Medicine guidelines recommended the continuous positive airway pressure as a first-line therapy for OSAS [14]. While continuous positive airway pressure is an established therapy for OSAS, adherence to it could be problematic because of adverse effects, such as dryness, sore throat, obstruction of nasal breathing, and discomfort [15]. In Japan, oral appliances (OAs) have been used as an alternative therapy for patients with mild-to-moderate OSAS ( $5 \leq$ Apnea-Hypopnea Index $[\mathrm{AHI}]<20$ ). However, some patients also experience adverse effects associated with OA therapy, including dental discomfort, joint pain, and dry mouth [16], which may affect compliance with OA use. One study reported that the discontinuation rate was $23.5 \%$ during a 1 -year treatment [17]. Given that OAs are the only available devices for the treatment of mild-to-moderate OSAS, other therapies are needed.

One review demonstrated the improvement of AHI scores with nasal trumpets [18]. While this review revealed success and tolerability after a short-term use, data demonstrating generalized use over long term are lacking. In addition, some patients felt discomfort because the nasal trumpets were thick and hard and, therefore, verifying long-term tolerance is important. A recent pilot study reported the efficacy of a new nasal trumpet, the nasal airway stent (NAS; Nastent ${ }^{\mathrm{TM}}$, 7 Dreamers Laboratories, Inc., Tokyo, Japan; shown in Fig. 1). The NAS consists of a tube-shaped shaft made from silicon rubber and a proximal nose clip to fix the device in place. The shaft does not collapse under conditions of normal breathing. The NAS was designed to improve breathing ability by insertion into a single nasal cavity. It is coated with hydrogel, as a lubricant, to ensure smooth insertion and is available in several lengths (120-145 mm) [19], enabling customized fitting for users. It is disposable, individually packaged, and sterilized. Among its positive characteristics are its extreme softness, small size, smooth insertion, and portability compared to other existing nasal trumpets. In a pilot study, NAS therapy decreased the AHI scores and oxygen desaturation; however, only a small number of participants were recruited [19]. We performed a prospective, single-arm interventional pilot study to evaluate whether NAS was useful and effective for the subjective symptom improvement of mild-tomoderate OSAS. 


\section{Materials and Methods}

\section{Patients}

This study was conducted from October 2015 to June 2017 at 4 healthcare sites in Japan (Keio University Hospital [Tokyo], RESM Shinyokohama Sleep \& Respiratory Medical Care Clinic [Yokohama], Kichijyouji Sleep Medical Clinic [Tokyo], and Shinjuku Sleep Medical Clinic [Tokyo]). Seventy-one participants diagnosed with mild-to-moderate OSAS ( $\leq \leq \mathrm{AHI}<20$ events/h) by overnight polysomnography (PSG) were recruited. The exclusion criteria were as follows: sleep $<3 \mathrm{~h}$ during treatment and $\mathrm{AHI}<5$ events/h on a portable home device before treatment. Patients with an index of $<5$ were excluded. We included patients if they were supine-position dependent. Supine-position dependence was defined in cases where the $\mathrm{AHI}$ in the supine sleeping position was at least 2 -fold the corresponding value in other sleeping positions. Moreover, patients with unmeasurable data due to inspection equipment troubles were excluded. We defined inconsistent NAS use because of patient intolerance (under 30\% usage in 1 month) as failures.

The study protocol (No. 20150080) was approved by the institutional review board of each participating institution, and all patients provided written informed consent. This study was registered in the UMIN Clinical Trials Registry as "The effectiveness of the nasal airway stent (Nastent ${ }^{\mathrm{TM}}$ ) in sleep apnea syndrome"; Identifier: UMIN000019423; URL: https://upload.umin.ac.jp/cgiopen-bin/ctr_e/ctr_view.cgi? recptno=R000021082.

\section{Study Design and Measurements}

The NAS was used as a treatment for OSAS and the patients inserted it into one of the narises; sleep-associated events were measured using a portable home device (WatchPAT200, Itamar Medical, Caesarea, Israel) [20]. The NAS was available in several sizes (120-145 $\mathrm{mm}$ ); therefore, the physicians decided regarding the appropriate length before treatment. The appropriate length was defined as the one in which the edge of the device was visible when the patients opened their mouths. Pharyngeal findings were evaluated simultaneously using the modified Mallampati classification [21], which has been used to screen patients with OSAS [22]. In this procedure, patients sit face to face with the physician with their mouth widely open to protrude their tongue. The airway is classified into 4 groups according to the structures seen by the physician [21]. Additionally, the participants completed the study questionnaires before treatment and at 1 month after the NAS use. The questionnaires included questions regarding their age, sex, BMI, and symptoms. The symptoms were evaluated using the 1997 version of the Epworth Sleepiness Scale (ESS) [23], the Japanese version of the Pittsburgh Sleep Quality Index (PSQI) [24], and the Hospital Anxiety and Depression Scale (HADS) [25]. The ESS, PSQI, and HADS cutoff values were $11,5.5$, and 8 , respectively. Additionally, the patients kept a daily diary during the treatment period and recorded the following information: (1) whether they used the NAS; (2) whether they felt discomfort; (3) whether they experienced dry mouth; (4) whether they felt a refreshed feeling in the morning; and (5) any additional comments. The patients used the WatchPAT200 before and immediately after the NAS treatment and after 1 month. The sleep hours, inspection time (the time which the patients wore the WatchPAT200), AHI (including supine and non-supine AHI), Respiratory Disturbance Index (RDI), Oxygen Desaturation Index (ODI), percutaneous oxygen satura- tion $\left(\mathrm{SpO}_{2}\right)$, heart rate, and snore volume were recorded by the WatchPAT200 device and collected at the completion of the study period.

We evaluated the NAS effects according to the measurements recorded by the WatchPAT200 and the questionnaire responses before and after treatment. We also evaluated differences in effects according to pharyngeal findings.

\section{Statistical Analysis}

Data are expressed as mean \pm standard deviation. The primary end point was the decrease in $\mathrm{AHI}$ after treatment; the secondary end points were the change in symptoms and the frequency of side effects. Differences in pre- and post-therapy AHI and other measurements were assessed using the Friedman, Greenhouse-Geisser, paired $t$, and Wilcoxon signed-rank tests, where appropriate. Subsequently, the participants were categorized into one of the 4 groups in accordance with the modified Mallampati score. Differences among the measurements in each group were evaluated using a 2-way repeated-measures ANOVA. Additionally, the preand post-treatment AHI scores in each group were evaluated using the paired $t$ test or Wilcoxon test, where appropriate. The sample size was calculated according to the results of a previous pilot study [19]. At an a level of 0.05 and power of $90 \%, 30$ individuals were required. As the dropouts were anticipated, 50 individuals were recruited. However, there were more dropouts than anticipated. Consequently, more individuals were recruited. All statistical analyses were performed using SPSS version 23 (IBM Corporation, Armonk, NY, USA).

\section{Results}

\section{Patient Characteristics}

There were 71 patients diagnosed with mild-to-moderate OSAS by overnight PSG who agreed to participate in this study. Fourteen patients dropped out due to personal reasons or were excluded because of technical difficulties, such as sensor and machine troubles while using the WatchPAT200, resulting in unmeasurable data. In addition, we excluded patients whose AHI scores were $<5$ based on the results of portable home devices before treatment; we also confirmed that the enrolled patients were not supine-position dependent. Therefore, 48 patients remained. Sixteen of them discontinued the use of the NAS during the first month due to discomfort and nasal mucus and were excluded from the study. We counted the response rates of all 48 initially enrolled. Finally, 32 patients completed the study and we assessed the effects of NAS on them (shown in Fig. 2). Their characteristics are summarized in Figure 3. The mean age and AHI score according to PSG was 52.7 years and 12.1 events/h, respectively. The mean PSQI scores were above the cutoff level of 5.5; however, the mean ESS and HADS scores were not (shown in Table 1). 


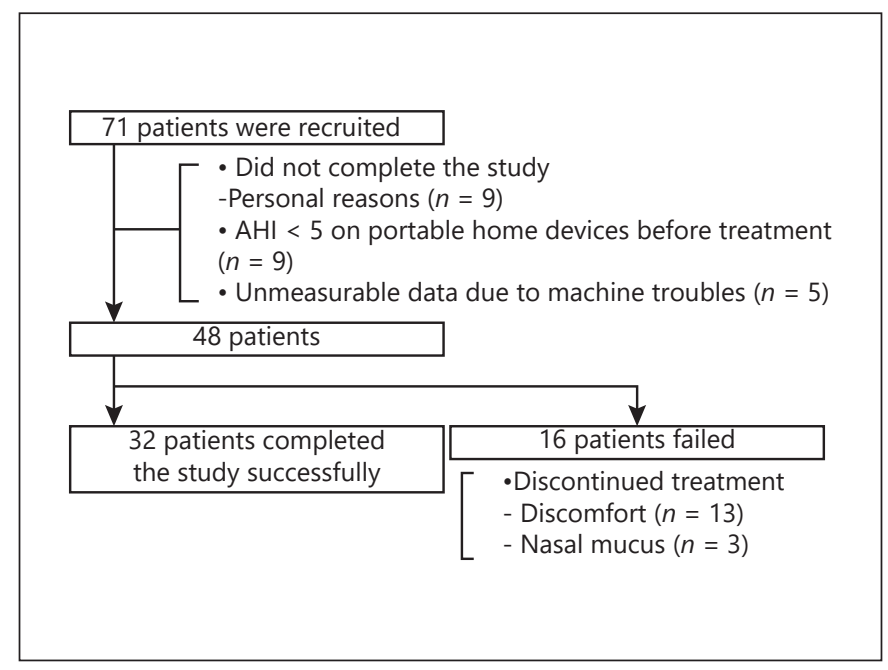

Fig. 2. Flow diagram of the study recruitment process.

Table 1. Baseline characteristics of the patients before treatment

\begin{tabular}{lc}
\hline Characteristic & Value \\
\hline$N(\mathrm{M} / \mathrm{F})$ & $32(21 / 11)$ \\
Age, years & $52.7 \pm 11.9$ \\
$\mathrm{BMI}, \mathrm{kg} / \mathrm{m}^{2}$ & $23.6 \pm 4.1$ \\
$\mathrm{AHI}, \mathrm{h}$ & $12.1 \pm 4.4$ \\
ESS & $7.0 \pm 4.0$ \\
PSQI & $6.0 \pm 2.9$ \\
HADS (anxiety) & $3.6 \pm 2.8$ \\
HADS (depression) & $5.4 \pm 3.5$
\end{tabular}

Data are presented as number (ratio) or mean \pm standard deviation. AHI, Apnea-Hypopnea Index; ESS, Epworth Sleepiness Scale; PSQI, Pittsburgh Sleep Quality; HADS, Hospital Anxiety and Depression Scale.

\section{Effectiveness of the NAS}

The post-treatment AHI scores and the corresponding after 1 month of treatment significantly improved (12.7-7.9, 8.6 events/h, respectively; $r=0.44, p<0.001)$ (shown in Fig. 3). In particular, the NAS improved the supine AHI (18.5-12.4, 10.8 events/h, respectively; $r=$ $0.48, p<0.001) ; 84.4 \%$ of patients were supine-position dependent (shown in Table 2). The success rate in this study was $60 \%$ (including the initially enrolled patients who were excluded due to failures and machine troubles). Subsequently, we defined the complete and partial responses, as the $\mathrm{AHI}$ scores reduced to $<5$ events/h and

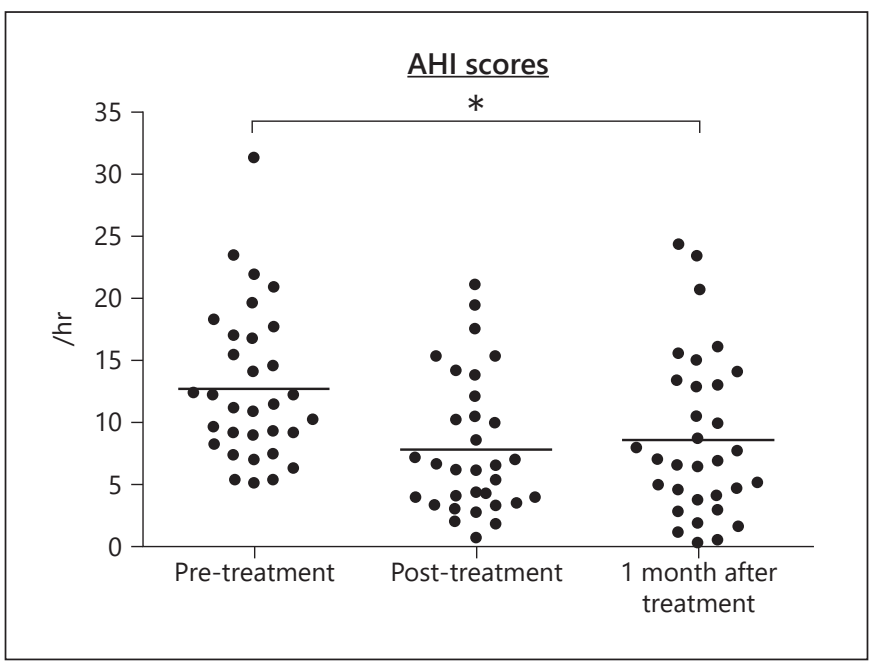

Fig. 3. The AHI scores before and after treatment. The NAS significantly improved the AHI scores immediately after and at 1 month after treatment. AHI, Apnea-Hypopnea Index; NAS, nasal airway stent.

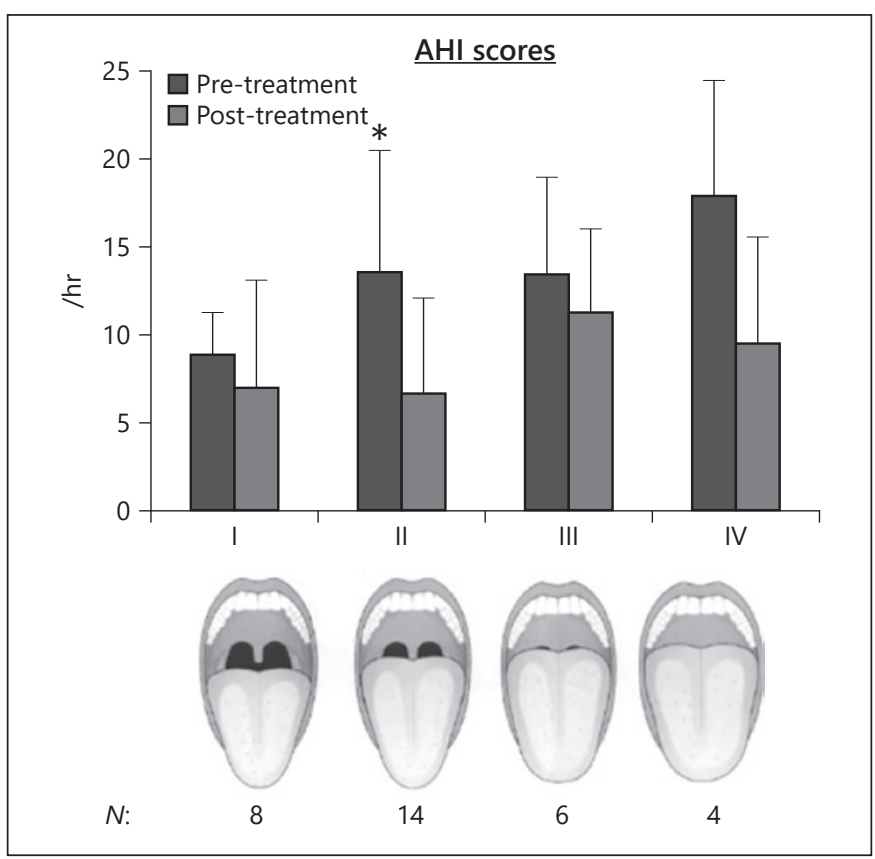

Fig. 4. Degree of improvement of the AHI scores in each group. Only patients with Mallampati class II exhibited the effects of the NSA in pre- and post-treatment comparisons. AHI, Apnea-Hypopnea Index; NAS, nasal airway stent.

by $>50 \%$, but still $>5$ events/h, respectively. The complete and partial response rates were 25.0 and $10.5 \%$, respectively. Additionally, the PAT-RDI and ODI scores improved $(r=0.3, p=0.011$ and $r=0.42, p<0.001$ for 
Table 2. Effectiveness of NAS therapy

\begin{tabular}{lcccc}
\hline & \multicolumn{2}{l}{ NAS therapy results } & \multirow{2}{*}{$p$ value } \\
\cline { 2 - 3 } & pre & post & after 1 month & \\
\hline Inspection time, min & $379.3 \pm 60.1$ & $391.6 \pm 73.1$ & $370.9 \pm 55.8$ & 0.298 \\
Sleeping hours, min & $330.6 \pm 53.4$ & $345.2 \pm 65.6$ & $324.2 \pm 51.0$ & 0.229 \\
PAT-RDI, /h & $17.2 \pm 5.8$ & $13.6 \pm 6.1$ & $14.0 \pm 6.8$ & 0.011 \\
3\% ODI, /h & $7.8 \pm 5.6$ & $4.4 \pm 4.1$ & $4.9 \pm 4.8$ & $<0.001$ \\
AHI, /h & $12.7 \pm 6.1$ & $7.9 \pm 5.5$ & $8.6 \pm 6.5$ & $<0.001$ \\
Supine AHI, /h & $18.5 \pm 12.2$ & $12.4 \pm 10.1$ & $10.8 \pm 8.7$ & $<0.001$ \\
Non-supine AHI, /h & $4.0 \pm 4.3$ & $3.8 \pm 4.2$ & $4.9 \pm 5.4$ & 0.467 \\
\% Time of supine sleep, \% & $61.6 \pm 27.8$ & $57.9 \pm 22.8$ & $81.8 \pm 11.6$ & 0.398 \\
Average SpO,$\%$ & $95.5 \pm 0.8$ & $95.7 \pm 0.9$ & $95.7 \pm 1.1$ & 0.247 \\
Minimum $\mathrm{SpO}, \%$ & $84.5 \pm 5.1$ & $88.2 \pm 3.5$ & $87.4 \pm 4.9$ & 0.002 \\
\% Time of $\mathrm{SpO}_{2}<90 \%, \%$ & $0.89 \pm 1.40$ & $0.30 \pm 0.60$ & $0.60 \pm 1.51$ & 0.077 \\
Minimum $\mathrm{HR}, /$ min & $46.0 \pm 5.5$ & $48.1 \pm 5.2$ & $44.6 \pm 6.0$ & 0.014 \\
Average $\mathrm{HR}, /$ min & $61.4 \pm 6.5$ & $62.3 \pm 7.4$ & $60.3 \pm 7.5$ & 0.044 \\
Average snore volume, dB & $45.0 \pm 4.2$ & $43.6 \pm 2.5$ & $43.4 \pm 2.6$ & 0.048 \\
\hline
\end{tabular}

Friedmann and Greenhouse-Geisser test for $p$ values. Data are presented as mean \pm standard deviation. NAS, nasal airway stent; RDI, Respirator Disturbance Index; ODI, Oxygen Desaturation Index; AHI, Apnea-Hypopnea Index; $\mathrm{SpO}_{2}$, percutaneous oxygen saturation.

the PAT-RDI and ODI scores, respectively). The ODI scores of 7 participants were $>10$ events/h. Among them, 5 showed improvement of ODI scores to $<10$ events/h after undergoing the NAS treatment. Furthermore, the NAS treatment resulted in increased $\mathrm{SpO}_{2}$, altered heart rate, and a significant improvement in the average snore volume (shown in Table 2). Furthermore, the percentage of time that a snore remained above $50 \mathrm{~dB}$ tended to be lower at post-treatment (13.4\%) and at 1 month after the treatment completion (13.2\%) compared to the corresponding values at pre-treatment $(21.8 \%)$ with $p=$ 0.045 and 0.095 , respectively (Wilcoxon signed-rank test).

Then, the participants were grouped according to the modified Mallampati classification. There were no statistically significant differences in age, BMI, or AHI scores among the 4 groups. The degree of AHI score improvement in each group was also evaluated. There were no significant differences among the 4 groups; however, preand post-treatment comparisons of patients with Mallampati class II showed that they benefitted from the NAS use (13.3-6.5 events/h; $p=0.012$ ) (shown in Fig. 4). The questionnaire responses reflected the NAS effects on patient symptoms. Although the post-treatment PSQI scores were significantly improved, there were no statistically significant differences in the ESS and HADS scores (shown in Table 3).

Nasal Airway Stent Efficiency for OSAS Patients
Table 3. Effectiveness of NAS therapy based on questionnaire responses

\begin{tabular}{llll}
\hline & \multicolumn{4}{l}{ NAS therapy results } \\
\cline { 2 - 4 } & pre & after 1 month & $p$ value \\
\hline ESS & $7.0 \pm 4.0$ & $6.8 \pm 4.4$ & 0.651 \\
PSQI & $6.0 \pm 2.9$ & $5.3 \pm 3.0$ & 0.022 \\
HADS (anxiety) & $3.6 \pm 2.8$ & $3.0 \pm 2.3$ & 0.119 \\
HADS (depression) & $5.4 \pm 3.5$ & $4.0 \pm 2.5$ & 0.210 \\
\hline
\end{tabular}

Wilcoxon, paired $t$ test for $p$ value. Data are presented as mean \pm standard deviation. NAS, nasal airway stent; ESS, Epworth Sleepiness Scale; PSQI, Pittsburgh Sleep Quality; HADS, Hospital Anxiety and Depression Scale.

\section{Adverse Events}

Discomfort was the most reported adverse effect of the NAS; $33.3 \%(n=16 / 48)$ of the patients could not use the NAS even for a few days. Similarly, $77.2 \%$ of patients who could use it consistently experienced discomfort to some extent, but this symptom gradually disappeared as they became accustomed to the use of the device. Finally, $29.4 \%$ of the patients had some lasting discomfort, but all patients used the NAS every day. Additionally, only 1 patient had a nasal mucosal trauma, observed by a small 
amount of blood at the top of the NAS. Thus, there were no severe enough adverse events to warrant consultation with a physician.

\section{Discussion}

Previous studies have shown that the NAS can reduce the AHI scores of patients without OSAS surgery $[19,26]$. However, these studies had a small number of participants and long-term effects were not assessed. In the present study, we demonstrated that NAS therapy decreased the AHI scores at 1 month after treatment. We found that the AHI was reduced from $12.7 / \mathrm{h}$ to $8.6 / \mathrm{h}$ at 1 month (approximately $33 \%$ reduction compared to the pre-AHI score). Further, we showed that the complete and partial response rates of the NAS were 25.0 and $10.8 \%$, respectively; the complete response rate was comparable with that of OAs [27-29].

One study has shown that ODI scores $>10$ events/h were associated with an increased risk of cardiovascular disease development [30]. In our study, the ODI scores of 7 participants were $>10$ events/h; 5 of these 7 participants exhibited improved ODI scores of $<10$ events/h after undergoing the NAS treatment, indicating that the latter may decrease the risk of developing cardiovascular diseases.

The Mallampati classification is an established method for assessing the pharynx status [31]. Interestingly, a previous study has used it to evaluate the NAS effect on the pharynx status [26]; therefore, we decided to use the Mallampati classification in the present study. We demonstrated that patients with Mallampati class II tended to show more improvements after using the NAS compared to the other Mallampati classification groups. However, we did not show the usefulness of the Mallampati classification as a predictive factor for the effectiveness of NAS therapy, probably because of the small number of patients included in each classification group; thus, more participants are needed to investigate the association between the Mallampati classification and the effectiveness of NAS therapy in future studies.

We found that NAS therapy significantly reduced the snore volume and the percentage of time, in which a snore remained above $50 \mathrm{~dB}$. This effect was immediate and it remained after 1 month. A decrease in higher-decibel snoring may provide better sleep quality. In contrast, only the PSQI scores improved. In Japan, the patients with severe OSAS often have low ESS scores; especially, the average score has been reported to be at 7.6 [32]. However, in this study, $40.6 \%$ of patients complained of sleepiness and $61.5 \%$ reported improved sleepiness after using the NAS. The low initial scores (ESS and HADS) before treatment may have led to a lesser improvement in these scores.

The side effects of NAS therapy included nasal mucus and discomfort; approximately $30 \%$ of the patients did not continue to use NAS therapy because of these side effects. Similarly, previous studies have reported that $23.5 \%$ of patients using OAs stopped the treatment as they faced tooth discomfort and periodontopathy [17]. In addition, only 1 person had nasal mucosal trauma during the 1-month use. This mucosal trauma was mild, with a small amount of blood observed on the NAS. The low incidence of mucosal trauma was likely due to the soft material of the NAS, suggesting that it could be used safely in the long-term.

Although our results revealed that there was only a small improvement in the AHI score after NAS use, it is important to note the improvement in subjective symptoms, such as sleepiness and the snore volume reduction. Our results showed that for some patients, the NAS worked as well as the OAs. Therefore, we propose that the NAS could be a potential treatment choice for patients with OSAS, because it is important to optimize treatment by trying and combining various methods [33].

There were several limitations in this study. First, the participants were limited to those with mild-to-moderate OSAS $(5 \leq \mathrm{AHI}<20)$ and the study population primarily comprised those with mild OSAS. This was lower than the range defined by the American Academy of Sleep Medicine (AASM) for cases of moderate OSAS (15-30). Additionally, the average AHI score was 12.1, which was higher than the recommended values for the mild category, as defined by the AASM standards. This may limit the generalization of our results to patients with moderate OSAS with higher AHI scores. Second, this study was not a randomized controlled trial. Rather, it was an uncontrolled study with a high dropout rate and was performed with the aim to justify future, controlled trials. Therefore, a randomized controlled trial would be necessary to establish the effectiveness of the NAS in patients with mild-tomoderate OSAS. Third, all the participants had BMIs within a healthy range according to the western BMI cutoff values. This may limit the generalizability of our results to overweight or obese patients. Fourth, this was a shortterm study; therefore, long-term adherence remains unknown. Further studies are needed to evaluate the effects of NAS therapy in patients with a wider OSAS severity and in obese patients, and to compare the effects between the NAS and OA therapies over a long-term period. 


\section{Conclusion}

Our results showed that NAS therapy might be effective in reducing symptoms and snoring in patients with mild-to-moderate OSAS. However, in this uncontrolled study, the response rate was found to be approximately one third and $30 \%$ of patients did not tolerate the NAS due to side effects. We believe that further clinical trials in patients with mild-to-moderate OSAS could lead to the recognition of the NAS as a new therapeutic device.

\section{Acknowledgements}

The authors wish to thank Maho Akiba and Yumi Hirata, joint researchers of Keio University School of Medicine, who contributed to this study.

\section{Statement of Ethics}

The study protocol (No. 20150080) was approved by the institutional review board of each participating institution, and the study was conducted ethically in accordance with the World Medical Association Declaration of Helsinki. All patients provided written informed consent prior to participation.

\section{Conflict of Interest Statement}

K.O., K.F., R.B., and T.B. were supported by funds donated by 7 Dreamers Laboratories, Inc. (Tokyo, Japan). However, this company had no role in the study design, data collection and analysis, or preparation of the manuscript. The other authors have no conflicts of interest to declare.

\section{Funding Sources}

This study received no funding.

\section{Author Contribution}

K. Fukunaga took full responsibility for the work, including the study design, access data, and the decision to submit and publish the manuscript. K. Otsuka was responsible for the study design, patient recruitment, data collection, data analysis, and writing of the manuscript. R. Baba, W. Yamasawa, R. Shirahama, Y. Hattori, and $\mathrm{H}$. Senoura were responsible for patient recruitment and data collection. T. Betsuyaku contributed to writing and discussion of the manuscript.

\section{References}

1 Owens RL, Eckert DJ, Yeh SY, Malhotra A. Upper airway function in the pathogenesis of obstructive sleep apnea: a review of the current literature. Curr Opin Pulm Med. 2008 Nov;14(6):519-24.

2 Dewan NA, Nieto FJ, Somers VK. Intermittent hypoxemia and OSA: implications for comorbidities. Chest. 2015 Jan;147(1):26674.

3 Subramanian S, Guntupalli B, Murugan T, Bopparaju S, Chanamolu S, Casturi L, et al. Gender and ethnic differences in prevalence of self-reported insomnia among patients with obstructive sleep apnea. Sleep Breath. $2011 \mathrm{Feb} ; 15(4): 711-5$.

4 Russell MB, Kristiansen HA, Kværner KJ. Headache in sleep apnea syndrome: epidemiology and pathophysiology. Cephalalgia. 2014 Sep;34(10):752-5.

5 Macey PM, Woo MA, Kumar R, Cross RL, Harper RM. Relationship between obstructive sleep apnea severity and sleep, depression and anxiety symptoms in newly-diagnosed patients. PLoS One. 2010 Apr;5(4):e10211.

6 Gilani S, Quan SF, Pynnonen MA, Shin JJ. Obstructive sleep apnea and gastroesophageal reflux: a multivariate population-level analysis. Otolaryngol Head Neck Surg. 2016 Feb; 154(2):390-5.

Nasal Airway Stent Efficiency for OSAS Patients
7 Fang HF, Miao NF, Chen CD, Sithole T, Chung MH. Risk of cancer in patients with insomnia, parasomnia, and obstructive sleep apnea: a nationwide nested case-control study. J Cancer. 2015 Sep;6(11):1140-7.

8 Gozal D, Ham SA, Mokhlesi B. Sleep apnea and cancer: analysis of a nationwide population sample. Sleep. 2016 Aug;39(8):1493-500.

9 Jonas DE, Amick HR, Feltner C, Weber RP, Arvanitis M, Stine A, et al. Screening for obstructive sleep apnea in adults: evidence report and systematic review for the US preventive services task force. JAMA. 2017 Jan; 317(4):415-33.

10 Marshall NS, Wong KK, Cullen SR, Knuiman MW, Grunstein RR. Sleep apnea and 20-year follow-up for all-cause mortality, stroke, and cancer incidence and mortality in the Busselton Health Study cohort. J Clin Sleep Med. 2014 Apr;10(4):355-62.

11 Punjabi NM, Caffo BS, Goodwin JL, Gottlieb DJ, Newman AB, O'Connor GT, et al. Sleepdisordered breathing and mortality: a prospective cohort study. PLoS Med. 2009 Aug; 6(8):e1000132.

12 Brenner R, Kivity S, Peker M, Reinhorn D, Keinan-Boker L, Silverman B, et al. Increased risk for cancer in young patients with severe obstructive sleep apnea. Respiration. 2019; 97(1):15-23.
13 Gaisl T, Baumgartner P, Rejmer P, Osswald M, Roeder M, Thiel S, et al. Prevalence of obstructive sleep apnea in patients with thoracic aortic aneurysm: a prospective, parallel cohort study. Respiration. 2020;99(1):19-27.

14 Kushida CA, Littner MR, Hirshkowitz M, Morgenthaler TI, Alessi CA, Bailey D, et al. Practice parameters for the use of continuous and bilevel positive airway pressure devices to treat adult patients with sleep-related breathing disorders. Sleep. 2006 Mar;29(3):375-80.

15 Weaver TE, Grunstein RR. Adherence to continuous positive airway pressure therapy: the challenge to effective treatment. Proc Am Thorac Soc. 2008 Feb;5(2):173-8.

16 Cistulli PA, Gotsopoulos H, Marklund M, Lowe AA. Treatment of snoring and obstructive sleep apnea with mandibular repositioning appliances. Sleep Med Rev. 2004 Dec;8(6): 443-57.

17 Shaun M, Rachael M, Victoria S, Victoria M, Michael B, William J, et al. Adherence and side effects among patients treated with oral appliance therapy for obstructive sleep apnea. J Dent Sleep Med. 2020 Apr;7(1).

18 Kumar AR, Guilleminault C, Certal V, Li D, Capasso R, Camacho M. Nasopharyngeal airway stenting devices for obstructive sleep apnoea: a systematic review and meta-analysis. J Laryngol Otol. 2015 Jan;129(1):2-10. 
19 Hirata Y, Satoh M. Pilot study of a nasal airway stent for the treatment on obstructive sleep apnea. J Sleep Disord Ther. 2015 Jul; 4(4):207.

20 Garg N, Rolle AJ, Lee TA, Prasad B. Homebased diagnosis of obstructive sleep apnea in an urban population. J Clin Sleep Med. 2014 Aug;10(8):879-85.

21 Avincsal MO, Dinc ME, Ulusoy S, Dalgic A, Ozdemir C, Develioglu ON. Modified Mallampati score improves specificity of STOPBANG questionnaire for obstructive sleep apnea. J Craniofac Surg. 2017 Jun;28(4):904-8.

22 Liistro G, Rombaux P, Belge C, Dury M, Aubert G, Rodenstein DO. High Mallampati score and nasal obstruction are associated risk factors for obstructive sleep apnoea. Eur Respir J. 2003 Feb;21(2):248-52.

23 Joan EB, Doerte UJ, Stefan S, John JP, Arthur AS. Pittsburgh and Epworth Sleep Scale Items: accuracy of ratings across different reporting periods. Behav Sleep Med. 2013; 11(3): 173-88

24 Doi Y, Minowa M, Uchiyama M, Okawa M, Kim K, Shibui K, et al. Psychometric assessment of subjective sleep quality using the Japanese version of the Pittsburgh Sleep Quality
Index (PSQI-J) in psychiatric disordered and control subjects. Psychiatry Res. 2000 Dec; 97(2-3):165-72.

25 Danni Z, Ying X, Shoujiang Y, Maree LH, Richard JW, Qiang L, et al. Effects of continuous positive airway pressure on depression and anxiety symptoms in patients with obstructive sleep apnoea: results from the sleep apnoea cardiovascular Endpoint randomised trial and meta-analysis. EClinicalMedicine. 2019 Jun;11:89-96.

26 Okuno K, Ono Minagi H, Ikai K, Matsumura Ai E, Takai E, Fukatsu H, et al. The efficacy of nasal airway stent (Nastent) on obstructive sleep apnoea and prediction of treatment outcomes. J Oral Rehabil. 2019 Jan;46(1):51-7.

27 Mehta A, Qian J, Petocz P, Darendeliler MA, Cistulli PA. A randomized, controlled study of a mandibular advancement splint for obstructive sleep apnea. Am J Respir Crit Care Med. 2001 May;163(6):1457-61.

28 Ng AT, Darendeliler MA, Petocz P, Cistulli PA. Cephalometry and prediction of oral appliance treatment outcome. Sleep Breath. 2012 Mar; 16(1):47-58.

29 Phillips CL, Grunstein RR, Darendeliler MA, Mihailidou AS, Srinivasan VK, Yee BJ, et al.
Health outcomes of continuous positive airway pressure versus oral appliance treatment for obstructive sleep apnea: a randomized controlled trial. Am J Respir Crit Care Med. 2013 Apr;187(8):879-87.

$30 \mathrm{Wu}$ WT, Tsai SS, Lin YJ, Lin MH, Wu TN, Shih TS, et al. Utility of overnight pulse oximeter as a screening tool for sleep apnea to assess the 8-year risk of cardiovascular disease: data from a large-scale bus driver cohort study. Int J Cardiol. 2016 Dec;225:206-12.

31 Nuckton TJ, Glidden DV, Browner WS, Claman DM. Physical examination: Mallampati score as an independent predictor of obstructive sleep apnea. Sleep. 2006 Jul;29(7):903-8.

32 Otsuka K, Fukunaga K, WakakoYamasawa, Haraguchi M, Tani T, Shirahama R, et al. Factors associated with improvements in subjective symptoms of obstructive sleep apnea syndrome after continuous positive airway pressure therapy. Sleep Breath. 2020 Jun;24(2): 491-8.

33 Vroegop AV, Vanderveken OM, Verbraecken JA. Drug-induced sleep endoscopy: evaluation of a selection tool for treatment modalities for obstructive sleep apnea. Respiration. 2020;99(5):451-7. 